\title{
Comparison of Diagnostic Efficacy among Transvaginal Sonography, Transabdominal Sonography, and 3.0 T Magnetic Resonance Imaging in Early Cesarean Scar Pregnancy
}

\author{
Ke Wang ${ }^{1}$ and Fangbin Jing $\mathbb{D}^{2}$ \\ ${ }^{1}$ Department of Ultrasound, Zibo First Hospital, Zibo 255200, Shandong Province, China \\ ${ }^{2}$ Department of Ultrasound Medicine, Laiyang Central Hospital of Yantai City, Yantai 265200, Shandong Province, China \\ Correspondence should be addressed to Fangbin Jing; jingfangbin@lyzxyy.org.cn
}

Received 10 December 2021; Revised 31 December 2021; Accepted 3 January 2022; Published 25 January 2022

Academic Editor: Bhagyaveni M.A

Copyright ( $) 2022$ Ke Wang and Fangbin Jing. This is an open access article distributed under the Creative Commons Attribution License, which permits unrestricted use, distribution, and reproduction in any medium, provided the original work is properly cited.

Objective. To compare the diagnostic efficacy among transvaginal sonography (TVS), transabdominal sonography (TAS), and 3.0 T magnetic resonance imaging (MRI) in early cesarean scar pregnancy (CSP). Methods. The clinical data of 65 patients initially diagnosed with CSP in our hospital from November 2019 to November 2020 were selected for the retrospective analysis, and all patients received TVS, TAS, and 3.0 T MRI. Taking the pathological findings as the "gold standard", the diagnostic efficacy of different diagnostic modalities in early CSP was compared. Results. In terms of the AUC value, the result was $3.0 \mathrm{~T}$ MRI > TVS > TAS, and among the three diagnosis methods, 3.0 T MRI had the highest diagnostic specificity, and TVS and 3.0 T MRI had the highest sensitivity; other than intragestational hemorrhage, the detection rates of other signs of disease by TVS and TAS were significantly higher than 3.0 T MRI $(P<0.05)$. Conclusion. 3.0 T MRI has better diagnostic efficacy in early CSP than TVS and TAS, while TVS and TAS work better in diagnosing uterine bleeding, plumule, yolk sac, and fetal heartbeat than $3.0 \mathrm{~T}$ MRI. The results are more beneficial to the guidance on selecting treatment modalities.

\section{Introduction}

Cesarean scar pregnancy (CSP) is a type of heterotopic pregnancy at a special site in pregnant women who have undergone cesarean section (C-section) before, which is mainly manifested by embryos implanted at the lower uterine segment where the incision scar is located and is one of the long-term complications of C-section [1,2]. The specific etiology remains unclear, and it is speculated that it may be related to the history of C-section or other surgery on the endometrium, which changes the environment of the local uterine cavity and results in the difficulty of the zygote to implant on the anterior and posterior walls of the uterine body, so the zygote migrates to the scar site for growth and development, and thereby leading to CSP. Recently, the incidence of CSP has increased year by year due to the increased rate of C-sections. Because the CSP has no specific manifestation, it is prone to clinical erroneous diagnosis and missed diagnosis, and failure to timely diagnose and take appropriate care measures will lead to major bleeding, uterine perforation, rupture, and other serious complications in patients [3-5]. At present, inquiring the medical history, imaging, and pathological examination are the common methods in clinic to confirm the diagnosis, and surgical exploration is a commonly used examination method in CSP, which is clinically regarded as the examination "gold standard", but it is risky and difficult to promote the application in primary hospitals. Therefore, imaging examination has an increasing role in diagnosing early CSP [6]. Ultrasonography is valuable for the diagnosis of CSP, which clearly shows the blood supply to the pregnant decidua from the uterine scar and indicates the embryo implantation site, but it is difficult for the diagnosis of atypical CSP because of its high operator requirement and 
inability to show the morphology of the gestational sac and its relationship with surrounding tissues, which may lead to missed diagnosis and erroneous diagnosis [7, 8]. Magnetic resonance imaging (MRI) technique has good contrast in soft tissue examination and is capable of multidirectional imaging, and its diagnostic efficacy has been demonstrated in early benign and malignant lesions of the breast, ovarian cancer, fibromas of the follicular membrane, and other diseases $[9,10]$.3.0 T MRI shows dynamic changes of human tissue structures more clearly because of its high imaging speed, high signal-to-noise ratio, and high image resolution, but such diagnostic modality has a relatively long scanning time and many artifacts. This study is expected to provide a more clinical basis for diagnosis and treatment of CSP by comparing the characteristics of transvaginal sonography (TVS), transabdominal sonography (TAS), and 3.0 T MRI in diagnosing early CSP and analyzing their advantages and disadvantages.

\section{Materials and Methods}

2.1. General Data. The clinical data of 65 patients initially diagnosed with CSP in our hospital from November 2019 to November 2020 were selected for the retrospective analysis, and the study met the World Medical Association Declaration of Helsinki (2013) [11]. Inclusion criteria: (1) patients had undergone more than one C-section before and were 22 to 45 years old, and their clinical symptoms included amenorrhea, an irregular small amount of vaginal bleeding, and mild abdominal pain; (2) 3-8 weeks after cessation of menstruation, elevated human chorionic gonadotropin $(\beta-H C G)$ value was found via blood examination; and (3) patients had completed clinical imaging data. Exclusion criteria: (1) puerpera had other types of heterotopic pregnancy (tubal pregnancy, abdominal pregnancy, cervical pregnancy, etc.); (2) patients were complicated with other gynecological diseases that affected the diagnosis of CSP; (3) patients had an allergic constitution and could not accept MRI; and (4) patients were complicated with reproductive system malignancies or had mental diseases.

2.2. Methods. The examination instrument selected was the DU8-M2 color Doppler ultrasonic tester (manufactured: Xuzhou Ruihua Electronic Science \& Technology Development Co., Ltd.), and the probe frequency for TVS and TAS was 5-9 MHz and 1-5 MHz, respectively. First, the TAS was performed. Patients filled their bladder before examination and then lied in spine position, coupling agent was applied to their lower abdomen, and the probe was placed on their abdomen for exploration to carefully observe whether the size and shape of the uterus, the presence or absence of abnormal adnexal mass, gestation sac in the uterine cavity, abdominal or pelvic hydrops, and echogenicity inside internal uterine cavity were associate with the uterine scar. After that, patients emptied their urine and received TVS examination in lithotomy position, an ultrasonic probe wrapped with a sterile condom was put into their cavity via vagina for full-range scanning, routine examination of the uterus, pelvic cavity, and bilateral appendixes, and observation of size, location, and echogenicity of gestation sac, to find out whether they were related to C-section scar $[6,12]$. Meanwhile, attention should be paid to observe whether there was a bulging mass or the presence or absence of a gestational sac at the incision of the uterine site, the specific location and morphology of the mass and gestational sac, and the trophoblast flow signal, and to measure the size of the gestational sac as well as the thickness of the muscular layer of the anterior uterine wall.

3.0 T MRI: patients were in spine position and asked to control their breathing; the MAGNETOM Skyra 3.0 T MRI superconducting scanner (manufactured: Siemens AG) and body phased-array coil was used to perform routine scanning with the following scanning sequences. Turbo spin echo (TSE): T1WI (TR $522 \mathrm{~ms}$, TE $21 \mathrm{~ms}$ ), T2WI (TR 3,700 ms, TE $93 \mathrm{~ms}$ ), slice thickness $5 \mathrm{~mm}$, slice gap $1.0 \mathrm{~mm}$, matrix $320 \times 320$, the field of view (FOV) $350 \mathrm{~mm} \times 350 \mathrm{~mm}$; fatsaturated T2WI sagittal sequence: TR 5,000 ms, TE $93 \mathrm{~ms}$, slice thickness $5 \mathrm{~mm}$, slice gap $1.0 \mathrm{~mm}$, matrix $320 \times 320$, and FOV $260 \mathrm{~mm} \times 260 \mathrm{~mm}$. The images were analyzed and observed by 2 experienced radiologists.

2.3. Observation Index. Taking the results of pathological diagnosis as the "gold standard," the diagnostic efficacy of TAS, TVS, and 3.0 T MRI examinations was compared.

2.4. Statistical Methods. The data obtained in this study were analyzed by SPSS 26.0 , the relevant accuracy rates of diagnosing CSP via 3 imaging modalities were examined by $\mathrm{X}^{2}$ test, and differences were considered statistically significant at $P<0.05$.

\section{Results}

3.1. Comparison of Results of Different Imaging Examinations and Pathological Diagnosis. The results of pathological diagnosis showed that 54 cases were positive and 11 cases were negative for CSP. For comparison of results of the three imaging diagnosis modalities and pathological diagnosis; see Tables 1-3.

3.2. Comparison of Diagnostic Efficacy among Different Imaging Diagnosis Modalities. The AUC values of diagnosing CSP via TAS, TVS, and $3.0 \mathrm{~T}$ MRI were $0.727,0.776$, and 0.844, respectively, and among them, 3.0 T MRI had the highest diagnostic specificity, and TVS and 3.0 T MRI had the highest sensitivity (Table 4).

\subsection{Comparison of Different Diagnosis Modalities in Detecting} Signs of Disease. Other than intragestational hemorrhage, the detection rates of other signs of disease by TVS and TAS were significantly higher than 3.0 T MRI $(P<0.05)$ Table 5.

3.4. Analysis of ROC Curves of Different Imaging Diagnosis Modalities. For analysis of ROC curves of the three imaging diagnosis modalities, see Figure 1. 
TABLE 1: Comparison of results of TAS and pathological diagnosis $(n)$.

\begin{tabular}{lcccc}
\hline TAS & \multicolumn{3}{c}{ Results of pathological diagnosis } & Total \\
& Positive & Negative & 3 & 50 \\
Positive & 47 & 8 & 15 \\
Negative & 7 & 11 & 65 \\
Total & 54 & 8 & \\
\hline
\end{tabular}

TABLE 2: Comparison of results of TVS and pathological diagnosis $(n)$.

\begin{tabular}{lcccc}
\hline TVS & \multicolumn{3}{c}{ Results of pathological diagnosis } & Total \\
& Positive & Negative & 51 \\
Positive & 49 & 2 & 14 \\
Negative & 5 & 11 & 65 \\
Total & 54 & 9 & 11 \\
\hline
\end{tabular}

TABle 3: Comparison of results of 3.0 T MRI and pathological diagnosis $(n)$.

\begin{tabular}{|c|c|c|c|}
\hline \multirow{2}{*}{ 3.0 T MRI } & \multicolumn{2}{|c|}{ Results of pathological diagnosis } & \multirow{2}{*}{ Total } \\
\hline & Positive & Negative & \\
\hline Positive & 50 & 2 & 52 \\
\hline Negative & 4 & 9 & 13 \\
\hline Total & 54 & 11 & 65 \\
\hline
\end{tabular}

TABLE 4: Comparison of diagnostic efficacy among different imaging diagnosis modalities.

\begin{tabular}{lcccr}
\hline Diagnosis modality & AUC value & Specificity & Sensitivity & S5\%CI \\
\hline TAS & 0.727 & 61.11 & 94.74 & $0.638-0.817$ \\
TVS & 0.776 & 68.75 & 96.43 & $0.693-0.861$ \\
3.0 T MRI & 0.844 & 73.33 & 96.43 & $0.768-0.920$ \\
\hline
\end{tabular}

TABLE 5: Comparison of results of different diagnosis modalities and pathological diagnosis [ $n$ (\%)].

\begin{tabular}{lccccc}
\hline Diagnosis modality & Uterine bleeding & Plumule & Yolk sac & Fetal heartbeat & Intragestational hemorrhage \\
\hline TAS & $33(84.62)^{*}$ & $21(84.00)^{*}$ & $25(83.33)^{*}$ & $12(70.59)^{*}$ & $24(66.67)^{*}$ \\
TVS & $35(89.74)^{* *}$ & $20(80.00)^{* *}$ & $24(80.00)^{* *}$ & $13(76.47)^{* *}$ & $22(61.11)^{* *}$ \\
3.0 T MRI & $6(15.38)$ & $5(20.00)$ & $7(23.33)$ & $2(11.76)$ & $32(88.89)$ \\
Pathological diagnosis & 39 & 25 & 30 & 17 & 36 \\
\hline
\end{tabular}

Note. ${ }^{*}$ Significant difference between TAS and 3.0 T MRI $(P<0.05) ;{ }^{* *}$ significant difference between TVS and 3.0 T MRI $(P<0.05)$.

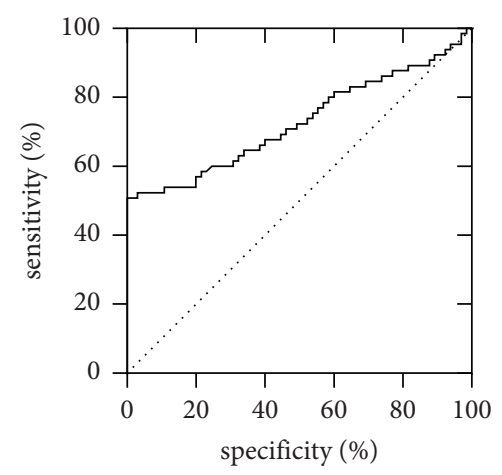

(a)

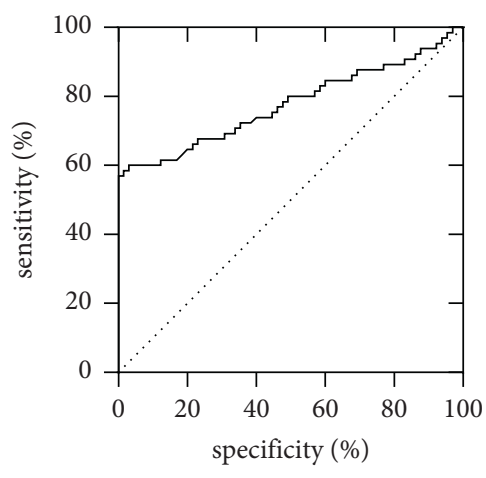

(b)

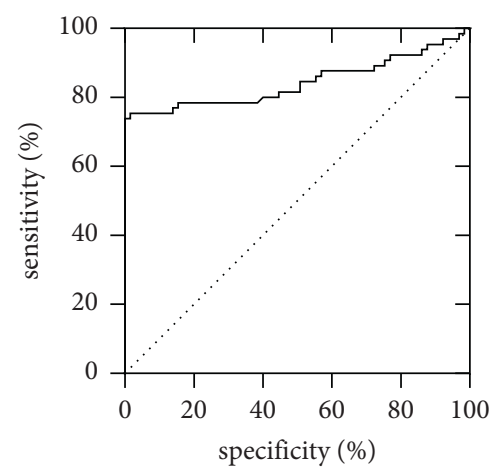

(c)

FIgUre 1: Analysis of ROC curves of different imaging diagnosis modalities. (a, b, c) the ROC curve of TAS, TVS, and 3.0 T MRI, respectively, diagnosing CSP; and the horizontal axes and vertical axes were the specificity (\%) and sensitivity (\%), respectively. 


\section{Discussion}

CSP is a special ectopic pregnancy manifested by the implantation of the gestational sac at the incision of a $\mathrm{C}$-section. At the early stage of CSP, there are no specific clinical manifestations, which largely increase the difficulty of diagnosis, and with the progress of pregnancy, the CSP will easily trigger uterine rupture, major bleeding, and other symptoms, seriously threatening the life safety of patients [13]. Ultrasound diagnosis is a common diagnostic modality for CSP, which launches an ultrasound beam to the human body to produce reflections at different demarcations of acoustic resistance in various tissues, and then the reflected echoes are accepted by the probe and then reconstituted into a sonogram for diagnosis. It is easy to perform, inexpensive and noninvasive, and the application of color Doppler ultrasound has obviously improved the reference value of ultrasound diagnosis [14]. The ultrasound diagnosis enables precise measurement of the physiological tissue structure and morphology in patients, with the diagnostic value that has been proven in diseases including adenomyosis and primary fallopian tube cancer [15]. The thickness of the muscle layer in the lower segment of the anterior uterine wall is currently a common index for the clinical diagnosis of CSP, which can fully reflect the damage situation at the uterine scar [16]. The TVA and TAS are two common modalities of ultrasound diagnosis to provide effective and important information for the treatment and prognosis of patients. With the development of ultrasound diagnosis technology, the TVA and TAS can detect early ectopic pregnancy and now have become the effective means to assist clinical diagnosis [5, 17, 18]. However, ultrasound diagnosis is susceptible to factors such as intestinal gas in patients and physicians' skill levels. MRI, a biological magnetic spin imaging technique, uses the characteristics of spin motion of the nucleus and generates a signal within the external magnetic field after being excited by radiofrequency pulses, then the signal is detected and entered into a computer for processing and transforming into images onscreen. As a multiplanar and multisequence imaging, the MRI has the characteristics such as high spatial resolution, high soft-tissue resolution, and high blood flow sensitivity, and can clearly show the location of the gestational sac in the lower uterine segment and accurately measure the thickness of the gestational sac and the anterior muscular layer, which has unique advantages in assessing the relationship with pelvic visceral structures and the depth of implanted scars [19].

The study results showed that compared with $3.0 \mathrm{~T}$ MRI, the AUC value was lower in TVS and TAS in diagnosing the CSP, demonstrating that $3.0 \mathrm{~T}$ MRI had higher diagnostic efficacy in confirming early CSP. Further analysis found that the AUC value of TVS was higher than that of TAS, and the reasons might be as follows: although the TAS was able to display the relationship between CSP position and the lower uterine segment as a whole and was convenient to observe the uterine corpus and cervix comprehensively, it confirmed the diagnosis using the principle of large interface reflection [20], hence it was vulnerable to factors such as bladder filling degree, abdominal wall thickness and intestinal gas, which led to sonography showing insignificant near-field echo, making it difficult to accurately measure the thickness between the bladder and the gestational sac, and thus resulting in missed diagnosis and erroneous diagnosis. In addition, compared with TAS, the TVS examination had significantly higher ultrasound probe frequency and higher resolution of the images, which, combined with the fact that the TVS could get more close to pelvic floor organs, would not be disturbed by some factors such as bowel gas and obesity, so the environment inside the uterus, the morphology, and location of the gestational sac, the blood flow signal, etc. could be observed clearly for making a more precise judgment [21]. Imaging and diagnosis with $3.0 \mathrm{~T} \mathrm{MRI}$ is a new imaging modality in recent years, which is widely used with the continuous advancement of technology and the continuous updating of equipment. Compared with ultrasound diagnosis, 3.0 T MRI enables full range, multiangle as well as multisequence imaging with higher resolution for soft tissue, accurate visualization of the location of the gestational sac, clearer visualization of the relationship between the lesion and the scar of C-section, presenting more obvious advantages in inspection and display for the tissues of pelvic organs [22]. On the other hand, 3.0 T MRI can also find the bleeding phenomenon in the uterine cavity at the local rupture of the myometrium, which is unparalleled by ultrasound diagnosis [23], thereby confirming that $3.0 \mathrm{~T}$ MRI has a high clinical application value in the diagnosis of early CSP. In addition, the diagnostic advantage of ultrasound was more prominent in the comparison with the pathological findings, which was due to the fact that 3.0 T MRI is more sensitive to the movement of body parts and prone to artifacts in diagnosis.

\section{Conclusion}

In conclusion, 3.0 T MRI has high diagnostic efficacy in early CSP diagnosis, and its effect is better than that of TVA and TAS, which is beneficial for physicians to carry out observation and develop treatment regimens, demonstrating its role in improving the prognostic effect. Shortcomings of this study: the included sample size was limited, and the findings still need more multicentered studies with large samples for further confirmation; in addition, the quantitative comparative analysis between the lesion and normal myometrium was not performed in this study, so it needs to be more deeply and meticulously explored in the future work.

\section{Data Availability}

The data used to support the findings of this study are available on reasonable request from the corresponding author.

\section{Conflicts of Interest}

The authors declare that there are no conflicts of interest. 


\section{References}

[1] I. E. Timor-Tritsch, A. Monteagudo, G. D’Antonio, and A. Kaelin Agten, "Cesarean scar pregnancy," Obstetrics \& Gynecology Clinics of North America, vol. 46, no. 4, pp. 797-811, 2019.

[2] F. Qi, Z.-Y. Chai, M.-M. Liu et al., "Type 2 cesarean scar pregnancy successfully treated via hysteroscopy-assisted laparoscopy," Journal of Minimally Invasive Gynecology, vol. 26, no. 7, pp. 1273-1281, 2019.

[3] L. Chen, S. Xiao, X. Zhu, S. He, and M. Xue, "Analysis of the reproductive outcome of patients with cesarean scar pregnancy treated by high-intensity focused ultrasound and uterine artery embolization: a retrospective cohort study," Journal of Minimally Invasive Gynecology, vol. 26, no. 5, pp. 883-890, 2019.

[4] Y. Wu, L. Zhou, Q. Zhou, and T. Zeng, "Efficacy of contrastenhanced ultrasound for diagnosis of cesarean scar pregnancy type," Medicine, vol. 98, no. 44, Article ID e17741, 2019.

[5] Q. Du, G. Liu, and W. Zhao, "A novel method for typing of cesarean scar pregnancy based on size of cesarean scar diverticulum and its significance in clinical decision-making," Journal of Obstetrics and Gynaecology Research, vol. 46, no. 5, pp. 707-714, 2020.

[6] D. Liu, X. Gu, F. Liu, F Shi, and M Yang, "Contrast-enhanced ultrasound in uterine artery embolization treatment of cesarean scar pregnancy," Journal of International Medical Research, vol. 48, no. 12, Article ID 300060520980217, 2020.

[7] X. Huo, Y. Xie, L. Yang, K. Deng, and Y. Liu, "Doppler ultrasound evaluation of the efficacy of high intensity focused ultrasound in treatment of cesarean section scar pregnancy," Journal of the College of Physicians and Surgeons Pakistan, vol. 30, no. 3, pp. 240-244, 2020.

[8] Z. Liu, Z. Shi, and Y. Dai, "The clinical and ultrasound-based comparison between cesarean scar pregnancy and other lower uterine segment pregnancies with a history of cesarean section," Journal of Maternal-Fetal and Neonatal Medicine, vol. 34, no. 4, pp. 639-645, 2021.

[9] S. Tsuji, A. Takahashi, A. Higuchi et al., "Pregnancy outcomes after hysteroscopic surgery in women with cesarean scar syndrome," PLoS One, vol. 15, no. 12, Article ID e0243421, 2020.

[10] X. Zhang, Y. Pang, Y. Ma et al., "A comparison between laparoscopy and hysteroscopy approach in treatment of cesarean scar pregnancy," Medicine, vol. 99, no. 43, Article ID e22845, 2020.

[11] World Medical Association, "World medical association declaration of helsinki: ethical principles for medical research involving human subjects," JAMA, vol. 310, no. 20, pp. 2191-2194, 2013.

[12] X. Xiong, C. Y. Gao, D. M. Ying et al., "Contrast-enhanced ultrasound evaluation of mifepristone for treatment of lowrisk cesarean scar pregnancy," Contrast Media and Molecular Imaging, vol. 2020, Article ID 3725353, 2020.

[13] E. A. van Wassenaer, F. A. E. de Voogd, R. R. van Rijn et al., "Diagnostic accuracy of transabdominal ultrasound in detecting intestinal inflammation in paediatric IBD patients-a systematic review," Journal of Crohn's and Colitis, vol. 13, no. 12, pp. 1501-1509, 2019.

[14] F. Moro, G. Bolomini, M. Sibal et al., "Imaging in gynecological disease (20): clinical and ultrasound characteristics of adnexal torsion," Ultrasound in Obstetrics and Gynecology, vol. 56, no. 6, pp. 934-943, 2020.
[15] Y. Gui, L. Wang, T. Gao, H Hong, and W Zhao, "Ultrasonic imaging combined with hysteroscopy in diagnosis of endometrial polyps based on multioperator algorithm and analysis of nerve growth factor receptor transmembrane protein expression," World neurosurgery, vol. 149, pp. 413-419, 2021.

[16] G. Masselli, M. Derme, M. G. Piccioni et al., "To evaluate the feasibility of magnetic resonance imaging in predicting unusual site ectopic pregnancy: a retrospective cohort study," European Radiology, vol. 28, pp. 2444-2454, 2018.

[17] X. Li, W. Sun, L. Chen et al., "Cesarean scar pregnancy combined with arteriovenous malformation successfully treated with transvaginal fertility-sparing surgery," Medicine, vol. 99, no. 31, Article ID e21432, 2020.

[18] W. Zhang and J. Chen, "Diffusion Tensor Imaging (DTI) of the cesarean-scarred uterus in vivo at 3T: comparison study of DTI parameters between nonpregnant and pregnant cases," Journal of Magnetic Resonance Imaging, vol. 51, no. 1, pp. 124-130, 2020.

[19] J. Hoffmann, M. Exner, K. Grothoff, P. Stumpp, and H. Stepan, "Comparison of the lower uterine segment in pregnant women with and without previous cesarean section in 3 T MRI," BMC Pregnancy and Childbirth, vol. 19, no. 1, p. $160,2019$.

[20] L. Pirtea, O. Balint, C. Grigoras, and R Ilina, "Laparoscopic resection of cesarean scar ectopic pregnancy after unsuccessful systemic methotrexate treatment," Journal of Minimally Invasive Gynecology, vol. 26, pp. 399-400, 2019.

[21] J. Fatusic, I. Hudic, A. Zildzic-Moralic, and B Hadziefendic, "Cesarean scar pregnancy complicated with placenta percreta," Medical Archives (Sarajevo, Bosnia and Herzegovina), vol. 73, pp. 58-60, 2019.

[22] M. A. Begam, H. Mirghani, W. Khair, H. Elbiss, T. Naeem, and S. M. Salahudeen, "Caesarean scar pregnancy: time to explore indications of the caesarean sections?" Journal of Obstetrics and Gynaecology, vol. 39, no. 3, pp. 365-371, 2019.

[23] M. M. Altay, S. A. Mert, A. Kaplan, and O. Gelisen, "Successful technique of manual vacuum aspiration for treatment of type 2 cesarean scar pregnancies: evaluation of 40 cases," Journal of Maternal-Fetal and Neonatal Medicine: The Official Journal of the European Association of Perinatal Medicine, the Federation of Asia and Oceania Perinatal Societies, the International Society of Perinatal Obstetricians, vol. 34, no. 16, pp. 2693-2700, 2021. 